\title{
Low-complexity Energy-efficient Resource Allocation for the Downlink of Cellular Systems
}

\author{
Fabien Héliot, Member, IEEE, Muhammad Ali Imran, Senior Member, IEEE, \\ and Rahim Tafazolli, Senior Member, IEEE
}

\begin{abstract}
Energy efficiency (EE) is undoubtedly an important criterion for designing power-limited systems, and yet in a context of global energy saving, its relevance for power-unlimited systems is steadily growing. Equally, resource allocation is a well-known method for improving the performance of cellular systems. In this paper, we propose an EE optimization framework for the downlink of planar cellular systems over frequency-selective channels. Relying on this framework, we design two novel lowcomplexity resource allocation algorithms for the single-cell and coordinated multi-cell scenarios, which are EE-optimal and EEsuboptimal, respectively. We then utilize our algorithms for comparing the EE performance of the classic non-coordinated, orthogonal and coordinated multi-cell approaches in realistic power and system settings. Our results show that coordination can be a simple and effective method for improving the $\mathrm{EE}$ of cellular systems, especially for medium to large cell sizes. Indeed, by using a coordinated rather than a non-coordinated resource allocation approach, the per-sector energy consumption and transmit power can be reduced by up to $15 \%$ and more than $90 \%$, respectively.
\end{abstract}

Index Terms-Energy efficiency, resource allocation, cellular system, multi-user, realistic power model.

\section{INTRODUCTION}

For decades, spectral efficiency (SE) has been one of the main criterion for designing reliable and scalable communication systems. In the current context of $\mathrm{CO}_{2}$ reduction and energy saving, energy efficiency (EE) is gradually becoming as important as SE for the development of future communication systems, e.g. LTE-advanced [1]. Although EE is nowadays a very trendy topic in communications [2]-[4], it is not by any means a new concept. Indeed, EE is already an important design criterion for power-limited as well as battery-driven systems and, consequently, many EE studies related to these topics can be found in the literature [5]-[7]. However, it still represents a new frontier for power-unlimited communication systems, such as cellular networks [8], [9]. This new interest in $\mathrm{EE}$ for power-unlimited systems can be explained by two factors; first, the ICT community aims at drastically decreasing its carbon footprint; second, network operators focus hard on reducing their operational costs. Adapting the energy consumption of such power-unlimited systems to their

Paper approved by A. Y. Zhang, the Editor for Optimization and Resource Allocation of the IEEE Communications Society. Manuscript received July 16, 2012; revised January 5, 2013; accepted March 13, 2013.

The authors are with the Centre for Communication Systems Research, Faculty of Electronics \& Physical Sciences, University of Surrey, Guildford GU2 7XH, UK (e-mail: F.Heliot@Surrey.ac.uk).

The research leading to these results has received funding from the European Commission's Seventh Framework Programme FP7/2007-2013 under grant agreement $\mathrm{n}^{\circ} 247733$-project EARTH. environment, i.e. channel conditions, user distributions, quality of service, etc., can be beneficial for meeting both these targets.

Such an adaptation can be performed through resource allocation and/or link adaptation by taking a full advantage of the channel state information. In the past, resource allocation has been extensively utilized for improving the SE or peak rate performance of communication systems [10], [11], but with little if any consideration about the energy consumption. With the growing importance of the EE as a system design criterion, EE-based allocation is gaining momentum over SEbased allocation [12]-[17]. When considering a transmission over a frequency selective channel in a single-cell scenario, or more generally the orthogonal multi-user channel (OMC), the SE-optimal power and rate allocation can simply be obtained through the classic water-filling algorithm [10], [18]. In order to obtain the EE-optimal resource allocation for the uplink of the OMC and, hence, saving user energy, the work in [13] proposed an iterative gradient search algorithm. This work assumed a linear power consumption model that served as a basis for its EE-based objective function. This work has then been revisited in [14] when considering a more complex power model. It has also recently been simplified and extended into a scheduling algorithm in [16]. Concerning the downlink of the OMC, a framework for optimizing the EE in an elastic traffic scenario has been introduced in [15]. Moreover, we have recently proposed an EE-based optimization framework for the broadcast channel in [17]. In a multi-cell context, multisite coordination and cooperation [19], [20] can be beneficial to mitigate or even take advantage of the interference in the downlink of cellular systems and, thus, improve the SE and/or EE performance. For instance in [21] and [22], [23], SE-based coordinated resource allocation strategies have been developed for multi-cell OFDM and OFDMA systems, respectively.

In this paper, we propose an EE optimization framework for the downlink of cellular systems over frequency-selective channel. Relying on this framework, we design two novel low-complexity EE-based resource allocation algorithms for the single-cell and coordinated multi-cell scenarios. In the single-cell scenario, we propose an EE-optimal algorithm for the downlink of the OMC by taking into account the total energy consumed within the cell rather than solely the users' power as in [13] and [14]. We prove the convexity of our objective function and derive the explicit formulations of the optimal users' power and rate for the unconstrained as well as power and rate constrained cases. In turn, we use these expressions for demonstrating that equal power allocation is 
both the most energy and spectral efficient strategy over the OMC when the channel gain-to-noise ratio is high. Moreover, our algorithm does not rely on a time-consuming iterative method as in [13]; its complexity is similar to that of the water-filling method. In the coordinated multi-cell scenario, we propose a suboptimal algorithm by taking into account the total energy consumed within a cluster of cells. We prove the convexity of our objective function and use some symmetry considerations on the cellular planar layout to come up with a low-complexity algorithm based on the Newton-Raphson method.

The rest of the paper is organized as follows. Section II describes the downlink cellular EE framework, i.e. the cellular system and power models, which is then used to formulate our energy-based objective functions, i.e. by considering the Jouleper-bit metric. In Section III, we propose our EE optimization framework and demonstrate that under certain assumptions both constrained and unconstrained EE optimization problems can be greatly simplified, i.e. these multivariate problems can be transformed into single variate problems. Based on this framework, we propose two novel EE-based resource allocation algorithms for the downlink of cellular systems in Section IV. We then utilize our algorithms for comparing the non-coordinated, orthogonal and coordinated multi-cell allocation approaches in realistic power and system settings. Our results indicate that coordination can be an effective method for improving the EE of cellular systems, in particular for medium to large cell sizes and when the main bulk of the users are close to their serving access point. Coordination can reduce the energy consumption and transmit power by up to $15 \%$ and more than $90 \%$, respectively, in comparison with the non-coordinated approach. A preliminary version of this paper is available in [24]. Herein, we have generalized our EE optimization framework to multi-cell systems, extended our single-cell resource allocation algorithm for the rate constrained case, proposed a novel energy-efficient algorithm for the coordinated multi-cell scenario, and compared our energyefficient strategies in a cellular layout.

\section{Cellular System EE Framework}

\section{A. System Model}

We consider the downlink of a planar cellular system, where sectorized base stations (BSs) with one antenna per sector communicate over a frequency-selective and block faded channel with user equipments (UEs) having a single antenna. More specifically, we consider a regular and symmetric cellular grid layout, as it is depicted in Fig. 1, where $\bar{M}$ BSs serve $K$ UEs spread over $M=3 \bar{M}$ sectors such that each sector contains $K_{m}$ users, with $K=\sum_{m=1}^{M} K_{m}$. Assuming that accurate channel state information is available at both BS and UE ends, the channel capacity per unit bandwidth of the $k$-th user within the $m$-th sector can be expressed as [25]

$$
\mathcal{C}_{m, k}=\log _{2}\left(1+\frac{g_{m, k}^{v(m)} p_{m, k}}{\Gamma\left(\sigma^{2}+I_{m, k}\right)}\right),
$$

where $g_{m, k}^{v(m)}$ is the channel gain between the $v(m)$-th BS and the $k$-th user of sector $m, v(m)=\lceil m / 3\rceil$ with $\lceil$.$\rceil being$

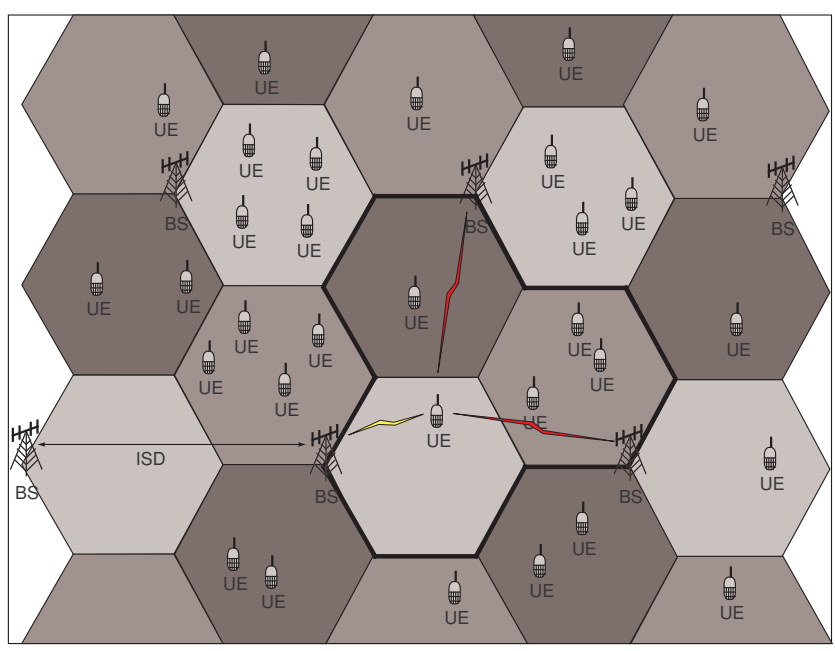

Fig. 1. Cellular system hexagonal layout.

the ceil operator, such that the $n$-th BS serves UEs in sectors $3 n-2,3 n-1$ and $3 n$. In addition, $\sigma^{2}$ is the noise power, $I_{m, k}$ accounts for the interference and $\Gamma$ represents the signal-tonoise ratio gap between the channel capacity and a practical coding and modulation scheme as in [13]. Moreover, $m \in$ $\mathcal{M}=\{1, \ldots, M\}$ and $k \in \mathcal{K}_{m}=\left\{1, \ldots, K_{m}\right\}$, where $\mathcal{M}$ and $\mathcal{K}_{m}$ are the sets of sector and user per sector indices, respectively. Conversely, the transmit power of the $k$-th user within the $m$-th sector, $p_{m, k}$, can be given based on (1) as

$$
p_{m, k}=\left(2^{\mathcal{C}_{m, k}}-1\right)\left(g_{m, k}^{v(m)}\right)^{-1} \Gamma\left(\sigma^{2}+I_{m, k}\right) .
$$

Consequently, the total transmit power and sum-rate of the system can be respectively given by

$$
\begin{aligned}
& P(\mathcal{C})=\Gamma \sum_{m=1}^{M} \sum_{k=1}^{K_{m}}\left(2^{\mathcal{C}_{m, k}}-1\right)\left(g_{m, k}^{v(m)}\right)^{-1}\left(\sigma^{2}+I_{m, k}\right) \text { and (3a) } \\
& R_{\Sigma}(\mathbf{P})=W \sum_{m=1}^{M} \sum_{k=1}^{K_{m}} \log _{2}\left(1+\frac{g_{m, k}^{v(m)} p_{m, k}}{\Gamma\left(\sigma^{2}+I_{m, k}\right)}\right),
\end{aligned}
$$

where $\mathbf{P}=\left[p_{1,1}, \ldots, p_{m, K_{m}}, \ldots, p_{M, K_{M}}\right] \succeq 0$ and $\mathcal{C}=$ $\left[\mathcal{C}_{1,1}, \ldots, \mathcal{C}_{m, K_{m}}, \ldots, \mathcal{C}_{M, K_{M}}\right] \succeq 0$.

\section{B. EE-SE trade-off and power consumption}

The existence of a trade-off between EE and SE [26] implies that EE and SE cannot be optimized separately. Indeed, in order to jointly optimize these two quantities, one has first to obtain its EE-SE trade-off explicit expression and use it as an objective function. In theory, the EE-SE trade-off of a point-topoint communication system consuming a total power of $P_{\Sigma}$ Watt for achieving a total rate of $R_{\Sigma}$ bit/s over a bandwidth $W(\mathrm{~Hz})$ can be formulated as [26]

$$
\frac{E_{b}}{N_{0}}=\frac{C^{-1}(\mathcal{C})}{\mathcal{C}},
$$

when only the radio frequency (RF) power of the transmitter is considered as consumed power, i.e. $P_{\Sigma}=P$. In addition, $E_{b}$ (Joule) is the transmitted energy per information bit, $N_{0}$ 
(Joule) is the noise spectral density, $\mathcal{C}$ is the channel capacity per unit bandwidth of the system and $C^{-1}(\mathcal{C})$ is its inverse function such that $C^{-1}(\mathcal{C})=P / \sigma^{2}$, where $\sigma^{2}=N_{0} W$.

In a traditional cellular system composed of BS and UE nodes, the total power consumption cannot be reduced only to the RF power. Notably, it has recently been indicated in various works [9], [27], [28] that the total consumed power of a BS accounts for various components such as a transceiver, power amplifier (PA), baseband interface, signal processing unit, power supply regulator and cooling system. These works have also shown that even though PAs are usually non-linear, the relation between the RF output power and BS total consumed power can be linearly abstracted as [9]

$$
P_{\mathrm{BS}}=\Delta_{\mathrm{BS}} P+t P_{\mathrm{BS}}^{C i},
$$

where $\Delta_{\mathrm{BS}}$ and $P_{\mathrm{BS}}^{C i}$ accounts for the RF dependent slope and circuit (fixed) power consumptions, respectively, and $t$ is the number of transmit antennas at the BS. In addition, the transmit power, i.e. RF output power, $P \in\left[0, t P_{\max }\right]$ with $P_{\max }$ being the maximum RF output power. As it has been indicated in [13], the total consumed power of an UE with one antenna can also be modeled via a linear relation, such that

$$
P_{\mathrm{UE}}=\Delta_{\mathrm{UE}} P+P_{\mathrm{UE}}^{C i} \text {. }
$$

Thus, the total power that is consumed by all the nodes in the cellular system for transmitting and receiving data can be linearly abstracted as

$$
P_{\Sigma}(\mathcal{C})=\Delta P(\mathcal{C})+P_{\mathrm{c}}
$$

when assuming the power models in (5) and (6) for all the BSs and UEs in the system, respectively. Moreover, $\Delta=\Delta_{\mathrm{BS}}, P_{\mathrm{c}}=P_{\mathrm{c}}(K)=M P_{\mathrm{BS}}^{C i}+\varsigma K P_{\mathrm{UE}}^{C i}$ or $\Delta=\Delta_{\mathrm{UE}}$, $P_{\mathrm{c}}=P_{\mathrm{c}}(K)=K P_{\mathrm{UE}}^{C i}+\varsigma M P_{\mathrm{BS}}^{C i}$ in the downlink or uplink scenario, respectively, where $\varsigma$ characterizes the ratio between transmission and reception overhead powers with $0 \leq \varsigma \leq 1$. Intuitively, less overhead power is necessary for receiving than for transmitting signals.

According to (4), the energy consumption, $E_{b}$, or EE, $1 / E_{b}$, is simply the ratio of the total consumed power to the sum-rate such that the EE-SE trade-off can be generalized as

$$
E_{b}=\left\{\begin{array}{l}
E_{b}(\mathcal{C})=\frac{\Delta P(\mathcal{C})+P_{\mathrm{c}}}{W \mathcal{C} 1^{T}} \text { or } \\
E_{b}(\mathbf{P})=\frac{\Delta \mathbf{P} 1^{T}+P_{\mathrm{c}}}{R_{\Sigma}(\mathbf{P})}
\end{array}\right.
$$

for the whole cellular system, where 1 is a $1 \times K$ vector of ones and $\{.\}^{T}$ is the transpose operator.

\section{EE OPTIMIZATION FRAMEWORK}

In this section, we first provide a framework for solving the unconstrained $\mathrm{EE}$ optimization problem in a simple manner by showing how to simplify it into a single variate problem under certain conditions. We then extend this framework to the power and rate constrained scenarios.

\section{A. Unconstrained Optimization}

Theorem 1: Assuming that:

1) $E_{b}$ in (8) is a convex function of $\mathcal{C}$ or $\mathbf{P}$;

2) The partial derivatives of each user interference are equal to each other, i.e. $\frac{\partial I_{m, k}}{\partial \mathcal{C}_{m, k}}=\frac{\partial I_{m, k}}{\partial \mathcal{C}_{n, l}}$ and $\frac{\partial I_{m, k}}{\partial p_{m, k}}=\frac{\partial I_{m, k}}{\partial p_{n, l}}$ for any $(m, n) \in \mathcal{M}^{2},(k, l) \in \mathcal{K}_{m}^{2}, m \neq n$ and $k \neq l$;

3 ) there is at least one active user in the system, e.g. the $l$-th user of sector $n$;

then the optimal unconstrained EE can be expressed solely as a function of the power or rate of the $l$-th user of sector $n$ such that

$E_{b}^{\star}=\left\{\begin{array}{l}\frac{\Delta P\left(\mathcal{C}_{n, l}^{\star}\right)+P_{\mathrm{c}}^{\star}}{W\left(K^{\star}\left[\mathcal{C}_{n, l}^{\star}+\log _{2}\left(\Gamma\left(g_{n, l}^{v(n)}\right)^{-1}\left(\sigma^{2}+I_{n, l}\right)\right)\right]-\beta\right)},(9 \mathrm{a}) \\ \frac{\Delta\left[K^{\star}\left(p_{n, l}^{\star}+\Gamma\left(g_{n, l}^{v(n)}\right)^{-1}\left(\sigma^{2}+I_{n, l}\right)\right)-\alpha\right]+P_{\mathrm{c}}^{\star}}{R_{\Sigma}\left(p_{n, l}^{\star}\right)},(9 \mathrm{~b})\end{array}\right.$

where

$$
\begin{aligned}
& P\left(\mathcal{C}_{n, l}^{\star}\right)=K^{\star} \Gamma\left(g_{n, l}^{v(n)}\right)^{-1}\left(\sigma^{2}+I_{n, l}\right) 2^{\mathcal{C}_{n, l}^{\star}-\alpha,} \\
& R_{\Sigma}\left(p_{n, l}^{\star}\right)=W\left(K ^ { \star } \operatorname { l o g } _ { 2 } \left(p_{n, l}^{\star}+\Gamma\left(g_{n, l}^{v(n)}\right)^{-1}\right.\right. \\
& \left.\left.\quad \times\left(\sigma^{2}+I_{n, l}\right)\right)-\beta\right), \\
& \alpha=\Gamma \sum_{m=1}^{M} \sum_{k \in \mathcal{K}_{m}^{\star}}\left(g_{m, k}^{v(m)}\right)^{-1}\left(\sigma^{2}+I_{m, k}\right) \quad \text { and } \\
& \beta=\sum_{m=1}^{M} \sum_{k \in \mathcal{K}_{m}^{\star}} \log _{2}\left(\Gamma\left(g_{m, k}^{v(m)}\right)^{-1}\left(\sigma^{2}+I_{m, k}\right)\right) .
\end{aligned}
$$

Moreover, $\mathcal{C}_{n, l}^{\star}$ and $p_{n, l}^{\star}$ are the optimal values of $\mathcal{C}_{n, l}$ and $p_{n, l}$, respectively, $P_{\mathrm{c}}^{\star}=P_{\mathrm{c}}\left(K^{\star}\right), K^{\star}$ is the optimal number of allocated users such that $K^{\star}=\sum_{m=1}^{M} K_{m}^{\star}$ with $1 \leq K_{m}^{\star} \leq K_{m}$ for any $m \in \mathcal{M}, K_{m}^{\star}=\left|\mathcal{K}_{m}^{\star}\right|$ and $\mathcal{K}_{m}^{\star}=\left\{k \in \mathcal{K}_{m} \mid \mathcal{C}_{m, k}^{\star}>0\right\}$ is the optimal set of per-sector allocated user indices.

In other words, under the three conditions listed above, the multivariate problem of finding the optimal unconstrained EE can be transformed into a single variate problem, which can then be solved in a straightforward manner by using a binary search algorithm [18] for finding $K^{\star}$. Note that equation (9) implicitly assumes the existence of at least one active user in the system, i.e. a user with a non-zero rate. Indeed, the energy consumption would go to infinity in (8) if no user is active in the system. Moreover, having sectors with no active user is not energy efficient either, and simple mechanisms can be used to disable temporarily such a sector [29], [30].

Proof: Assuming that $E_{b}$ is a convex function, it implies that there exists a unique $\mathcal{C}$ and $\mathbf{P}$ for which $E_{b}$ is optimal over its entire domain. Let $\mathcal{C}^{\star}$ and $\mathbf{P}^{\star}$ be these optimum values of $\mathcal{C}$ and $\mathbf{P}$, respectively, they then satisfy $\nabla E_{b}\left(\mathcal{C}^{\star}\right)=\mathbf{0}$ and $\nabla E_{b}\left(\mathbf{P}^{\star}\right)=\mathbf{0}$ such that $\frac{\partial E_{b}\left(\mathcal{C}^{\star}\right)}{\partial \mathcal{C}_{m, k}}=$ $\frac{\Delta}{W \mathcal{C}^{\star} \mathbf{1}^{T}} \frac{\partial P\left(\mathcal{C}^{\star}\right)}{\partial \mathcal{C}_{m, k}}-\frac{W P_{\Sigma}\left(\mathcal{C}^{\star}\right)}{\left(W \mathcal{C}^{\star} \mathbf{1}^{T}\right)^{2}}=0$ and $\frac{\partial E_{b}\left(\mathbf{P}^{\star}\right)}{\partial p_{m, k}}=\frac{\Delta}{R_{\Sigma}\left(\mathbf{P}^{\star}\right)}-$ 
$\frac{\Delta \mathbf{P}^{\star} \mathbf{1}^{T}+P_{\mathrm{c}}^{\star}}{R_{\Sigma}\left(\mathbf{P}^{\star}\right)^{2}} \frac{\partial R_{\Sigma}\left(\mathbf{P}^{\star}\right)}{\partial p_{m, k}}=0$. In turn, it implies that

$$
\begin{aligned}
& E_{b}^{\star}=E_{b}\left(\mathcal{C}^{\star}\right)=\frac{\Delta}{W} \frac{\partial P\left(\mathcal{C}^{\star}\right)}{\partial \mathcal{C}_{m, k}} \quad \text { and } \\
& \frac{1}{E_{b}^{\star}}=\frac{1}{E_{b}\left(\mathbf{P}^{\star}\right)}=\frac{1}{\Delta} \frac{\partial R_{\Sigma}\left(\mathbf{P}^{\star}\right)}{\partial p_{m, k}}
\end{aligned}
$$

for any $m \in \mathcal{M}$ and $k \in \mathcal{K}_{m}^{\star}$. Relying on the definition of $P(\mathcal{C})$ in $(3 \mathrm{a}), \frac{\partial P\left(\mathcal{C}^{\star}\right)}{\partial \mathcal{C}_{m, k}}=\frac{\partial P\left(\mathcal{C}^{\star}\right)}{\partial \mathcal{C}_{n, l}}$ is equivalent to

$$
\begin{aligned}
& \sum_{i=1}^{M} \sum_{j \in K_{i}^{\star}} \frac{\partial I_{i, j}}{\partial \mathcal{C}_{m, k}}\left(g_{i, j}^{v(i)}\right)^{-1}\left(2^{\mathcal{C}_{i, j}^{\star}}-1\right)+\ln (2)\left(g_{m, k}^{v(m)}\right)^{-1} \\
& \times 2^{\mathcal{C}_{m, k}^{\star}}\left(\sigma^{2}+I_{m, k}\right)=\ln (2)\left(g_{n, l}^{v(n)}\right)^{-1} 2^{\mathcal{C}_{n, l}^{\star}}\left(\sigma^{2}+I_{n, l}\right) \\
& +\sum_{i=1}^{M} \sum_{j \in K_{i}^{\star}} \frac{\partial I_{i, j}}{\partial \mathcal{C}_{n, l}}\left(g_{i, j}^{v(i)}\right)^{-1}\left(2^{\mathcal{C}_{i, j}^{\star}}-1\right),
\end{aligned}
$$

which yields the following relation between $\mathcal{C}_{n, l}^{\star}$ and $\mathcal{C}_{m, k}^{\star}$

$$
\mathcal{C}_{m, k}^{\star}=\mathcal{C}_{n, l}^{\star}+\log _{2}\left(\frac{g_{m, k}^{v(m)}\left(\sigma^{2}+I_{n, l}\right)}{g_{n, l}^{v(n)}\left(\sigma^{2}+I_{m, k}\right)}\right)
$$

if $\frac{\partial I_{m, k}}{\partial \mathcal{C}_{m, k}}=\frac{\partial I_{m, k}}{\partial \mathcal{C}_{n, l}}$ for any $(m, n) \in \mathcal{M}^{2},(k, l) \in \mathcal{K}_{m}^{\star^{2}}, m \neq n$ and $k \neq l$. Similarly, relying on the definition of $R_{\Sigma}(\mathbf{P})$ in (3b), $\frac{\partial R_{\Sigma}\left(\mathbf{P}^{\star}\right)}{\partial p_{m, k}}=\frac{\partial R_{\Sigma}\left(\mathbf{P}^{\star}\right)}{\partial p_{n, l}}$ yields

$p_{m, k}^{\star}=p_{n, l}^{\star}+\Gamma\left[\left(g_{n, l}^{v(n)}\right)^{-1}\left(\sigma^{2}+I_{n, l}\right)-\left(g_{m, k}^{v(m)}\right)^{-1}\left(\sigma^{2}+I_{m, k}\right)\right]$

if $\frac{\partial I_{m, k}}{\partial p_{m, k}}=\frac{\partial I_{m, k}}{\partial p_{n, l}}$ for any $(m, n) \in \mathcal{M}^{2}(k, l) \in \mathcal{K}_{m}^{\star^{2}}, m \neq n$ and $k \neq l$. We then obtain that $P\left(\mathcal{C}^{\star}\right)=P\left(\mathcal{C}_{n, l}^{\star}\right)$ in (10a) and $R_{\Sigma}\left(\mathbf{P}^{\star}\right)=R_{\Sigma}\left(p_{n, l}^{\star}\right)$ in (10b) by inserting (12) and (13) into (3a) and (3b), respectively. Next, equations (9a) and (9b) are obtained by inserting the latter results as well as (12) and (13) into (8a) and (8b), respectively.

\section{B. Constrained Optimization}

Thus far, we have shown that the EE optimization problem can be greatly simplified in the unconstrained case; in the following, we discuss the power and rate constrained cases.

Assuming a per-sector transmit power constraint, the EE optimization problem is given by

$$
\begin{array}{ll}
\min _{\mathbf{P}} & E_{b}(\mathbf{P}) \\
\text { s.t. } & \mathbf{P} \succeq 0, \mathbf{P}_{m} \mathbf{1}^{T} \leq P_{\max }, \forall m \in \mathcal{M},
\end{array}
$$

where $\mathbf{P}_{m}=\left[\mathbf{0}^{1 \times \varphi_{m}}, p_{m, 1}, \ldots, p_{m, K_{m}}, \mathbf{0}^{1 \times\left(K-\varphi_{m}-K_{m}\right)}\right]$ is the per-sector vector of transmit power, $\varphi_{m}=\sum_{i=1}^{m-1} K_{i}$ and $0^{1 \times \varphi_{m}}$ is a $1 \times \varphi_{m}$ vector of zeros. As long as the unconstrained optimal per-sector total power, $\mathbf{P}_{m}^{\star} \mathbf{1}^{T}$, is below $P_{\max }$ for any sector, then the EE optimization problem in (14) is equivalent to the unconstrained problem, which can be possibly solved by using (9b). At the other extreme, if all the antennas of all the BSs transmit at full power then $\mathbf{P} 1^{T}=M P_{\max }$ in $(8 \mathrm{~b})$ and $E_{b}(\mathbf{P})$ is equivalent to $\frac{M \Delta P_{\max }+P_{\mathrm{c}}}{R_{\Sigma}(\mathbf{P})}$ such that (14) reverts to maximizing $R_{\Sigma}(\mathbf{P})$ subject to $\mathbf{P} \mathbf{1}^{T}=M P_{\max }$, i.e. a classic $\mathrm{SE}$ maximization problem, which can solved via the water-filling method in absence of interference (see example 5.2 of [18]). In the first stage of the method, Karush-Kuhn-Tucker (KKT) conditions are utilized to obtain the following equality

$$
\frac{\partial R_{\Sigma}\left(\mathbf{P}^{\star}\right)}{\partial p_{m, k}}=\nu^{\star}
$$

where $\frac{\partial R_{\Sigma}(\mathbf{P})}{\partial p_{m, k}}=W\left[\ln (2)\left(p_{m, k}+\Gamma \sigma^{2}\left(g_{m, k}^{v(m)}\right)^{-1}\right)\right]^{-1}$ in absence of interference and $\nu^{\star}$ is the Lagrange multiplier for the equality constraint. Consequently, the optimum per-user power allocation is given by

$$
p_{m, k}^{\star}=\left[W\left(\ln (2) \nu^{\star}\right)^{-1}-\Gamma \sigma^{2}\left(g_{m, k}^{v(m)}\right)^{-1}\right]_{+},
$$

where $\left(\nu^{\star}\right)^{-1}$ represents the water-level and $[x]_{+}=$ $\max \{x, 0\}$. Comparing equations (15) with (11b) indicates that the unconstrained EE optimization problem can be solved in a similar manner as in (16), but where $\left(\nu^{\star}\right)^{-1}=E_{b}^{\star} \Delta^{-1}$ such that $E_{b}^{\star} \Delta^{-1}$ acts as a water-level.

Similarly, when assuming a per-sector sum-rate constraint, the EE optimization problem is given by

$$
\begin{array}{ll}
\min _{\mathcal{C}} & E_{b}(\mathcal{C}) \\
\text { s.t. } & \mathcal{C} \succeq 0, W \mathcal{C}_{m} \mathbf{1}^{T} \geq R_{\min }, \forall m \in \mathcal{M},
\end{array}
$$

where $\mathcal{C}_{m}=\left[\mathbf{0}^{1 \times \varphi_{m}}, \mathcal{C}_{m, 1}, \ldots, \mathcal{C}_{m, K_{m}}, \mathbf{0}^{1 \times\left(M-\varphi_{m}-K_{m}\right)}\right]$ is the per-sector vector of channel capacity per unit bandwidth. Following the same analysis as in the power-constrained case, as long as $W \mathcal{C}_{m}^{\star} \mathbf{1}^{T}$ is above $R_{\min }$ for any sector then (17) is equivalent to the unconstrained problem. However, in the case that all the sectors achieve only the minimum sumrate, $W \mathcal{C} 1^{T}=M R_{\min }$ in $(8 \mathrm{a})$ and $E_{b}(\mathcal{C})$ is equivalent to $\frac{\Delta P(\mathcal{C})+P_{\mathrm{c}}}{M R_{\min }}$ such that the optimization problem in (17) reverts to minimizing $P(\mathcal{C})$ subject to $W \mathcal{C}^{T}=M R_{\text {min }}$, i.e. a classic power minimization problem, which can also be solved via water-filling in absence of interference.

The EE optimization problem becomes either a joint EE/rate or EE/power problem when either some antennas transmit at less than full power or some sectors achieve more than the minimum sum-rate, respectively. Thus, EE optimization is a generalization of both SE and power optimizations such that the global optimal EE solution is the optimal unconstrained EE solution. Enforcing rate or power constraints on EE can provide either a SE or power optimal solution, which is however only suboptimal in terms of EE. In other words, in an ideal energy-efficient system, no antennas should transmit at full power for meeting and keeping quality of service.

\section{USE CASE SCENARIOS}

In the previous section, we proposed a generic EE optimization framework for the downlink of cellular systems. We apply it here for designing low-complexity EE-based resource allocation algorithms in two specific scenarios: single-sector/cell with no interference and multi-sector/cell coordination.

\section{A. Single-cell EE optimization}

Optimizing the EE of the whole cellular system requires that each BS knows the channel gain and interference of all the 
links in the system, which is not a realistic assumption. A more realistic assumption would be to consider that each BS knows only the channel gains of its own cell users and optimizes its $\mathrm{EE}$ as if each cell was insulated from the others. Note that by design each sector of a cell is insulated from the others and, hence, the optimization can be carried out on a per-sector basis, i.e. equivalent to a single cell with an omnidirectional antenna. Assuming that $M=1, K=K_{1}$ and $I_{1, k}=0$ for any $k \in \mathcal{K}=\mathcal{K}_{1}$ in (3b), the per-sector sum-rate simplifies to

$$
R_{\Sigma}(\mathbf{P})=W \sum_{k=1}^{K} \log _{2}\left(1+\frac{g_{k} p_{k}}{\Gamma \sigma^{2}}\right)
$$

which corresponds for instance to the sum-rate of a pointto-point transmission over a closed-loop multi-input multioutput channel with $K$ eigenmodes as well as of a $K$-user OFDMA transmission over a frequency-selective and block faded channel. We refer this scenario as OMC scenario.

1) Convexity of $E_{b}$ : Since $I_{1, k}=0$ for any $k \in \mathcal{K}$, then assumption 2) of Theorem I is clearly satisfied. Moreover, $E_{b}$ in (8a) simplifies to

$$
E_{b}(\mathcal{C})=A f(\mathcal{C}) g(\mathcal{C})^{-1}
$$

in the OMC scenario, where

$$
f(\mathcal{C})=B+\sum_{k=1}^{K}\left(2^{\mathcal{C}_{k}}-1\right) g_{k}^{-1} \text { and } g(\mathcal{C})=\sum_{k=1}^{K} \mathcal{C}_{k}
$$

In addition, $A=W^{-1} \Gamma \sigma^{2} \Delta$ and $B=\frac{P_{\mathrm{c}}}{\Gamma \sigma^{2} \Delta}$. The function $g(\mathcal{C})^{-1}$ is log-convex if $\mathcal{C}_{l}>0$, i.e. at least one user is active in the sector/cell; whereas, $f(\mathcal{C})$ is log-convex for $\mathcal{C}_{l}>0$ and $P_{c} \geq \Delta \alpha$, as it is proved in the Appendix. Hence, $E_{b}(\mathcal{C})$ in (19) is a log-convex and, thus, convex function such that assumption 1) of Theorem I is satisfied.

2) Unconstrained EE optimization: Since equality (11a) holds in the OMC scenario, it yields

$$
E_{b}^{\star}=A \ln (2) g_{l}^{-1} 2^{\mathcal{C}_{l}^{\star}} .
$$

Inserting (21) in the left side of equality (9a) allows us to reexpressed the latter solely as a function of $\mathcal{C}_{l}^{\star}$, such that the $l$-th user unconstrained EE-optimal channel capacity per unit bandwidth over the OMC can explicitly expressed as

$\mathcal{C}_{l}^{\star}=\frac{1}{\ln (2)}\left[W_{0}\left(\frac{\left(P_{c}^{\star}-\Delta \alpha\right) \chi e^{-1}}{K^{\star} \Gamma \sigma^{2} \Delta}\right)+1\right]+\log _{2}\left(\frac{g_{l}}{\Gamma \sigma^{2}}\right)+\frac{\beta}{K^{\star}}$,

where $\chi=\Gamma \sigma^{2} 2^{-\beta / K^{\star}}=\left(\prod_{k \in \mathcal{K}^{\star}} g_{k}\right)^{1 / K^{\star}}$ and $W_{0}$ denotes the real branch of the Lambert function [31]. Following the same process but using (11b) and (9b) instead of (11a) and (9a), the $l$-th user unconstrained EE-optimal transmit power can be explicitly formulated as

$$
p_{l}^{\star}=e^{W_{0}\left(\frac{\left(P_{c}^{\star}-\Delta \alpha\right) \chi e^{-1}}{K^{\star} \Gamma \sigma^{2} \Delta}\right)+1+\frac{\ln (2) \beta}{K^{\star}}}-\Gamma \sigma^{2} g_{l}^{-1} .
$$

3) Power constrained EE optimization: In the case that the optimal unconstrained transmit power is equal or greater than $P_{\text {max }}$, the optimal constrained transmit power then becomes $P_{\max }$, such that $P\left(\mathcal{C}^{\star}\right)=P_{\max }$. Consequently, (10a) can be re-expressed as $P_{\max }=K^{\star} \Gamma \sigma^{2} g_{l}^{-1} 2^{\mathcal{C}_{l}^{\star}}-\alpha$ for $M=1, K=$ $K_{1}$ and $I_{1, k}=0, \forall k \in \mathcal{K}$, which in turn implies that the $l$-th user power constrained EE-optimal channel capacity per unit bandwidth is given by

$$
\mathcal{C}_{l}^{\star}=\log _{2}\left(g_{l}\left(P_{\max }+\alpha\right)\right)-\log 2\left(K^{\star} \Gamma \sigma^{2}\right) .
$$

4) Rate constrained EE optimization: In the case that the optimal unconstrained sum-rate is equal or lower than $R_{\text {min }}$, then $R_{\Sigma}\left(\mathbf{P}^{\star}\right)=R_{\text {min }}$. Consequently, (10b) can be reexpressed as $R_{\min }=W\left(K^{\star} \log _{2}\left(p_{l}^{\star}+\Gamma \sigma^{2} g_{l}^{-1}\right)-\beta\right)$, which in turn implies that the $l$-th user rate constrained EE-optimal transmit power is given by

$$
p_{l}^{\star}=2^{\frac{1}{K^{\star}}\left(\frac{R_{\min }}{W}+\beta\right)}-\Gamma \sigma^{2} g_{l}^{-1} .
$$

5) EE optimization algorithm: In order to obtain the optimal unconstrained or constrained $\mathcal{C}_{l}^{\star}$ and $p_{l}^{\star}$ via the explicit expressions in (22), (23), (24) and (25), $\mathcal{K}^{\star}$ has to be known. Let $\pi$ be the user index order, with $\pi=\left(\pi_{1}, \ldots, \pi_{K}\right)$ denotes a permutation of $\mathcal{K}$, such that user $\pi_{1}$ and $\pi_{K}$ are the users with the largest and smallest channel gains, respectively. Given that at least one user is active in the cell, it leads to $\mathcal{C}_{\pi_{1}}>0$ as well as $\mathcal{C}_{\pi_{1}}^{\star}>0$ with $\pi_{1} \in \mathcal{K}^{\star}$ and, hence, $K^{\star} \geq 1$. Moreover, the following inequality

$$
\frac{P_{c}(U)}{\Gamma \sigma^{2} \Delta}>\sum_{k=1}^{U} g_{\pi_{k}}^{-1}-U\left(\prod_{k=1}^{U} g_{\pi_{k}}\right)^{-\frac{1}{U}},
$$

which is a direct consequence of the fact that the domain of $W_{0}$ is lower bounded by $-e^{-1}$, can be used for obtaining $\mathcal{K}^{\star}$. Starting from $U=K$ and decrementing $U$ by 1 as long as the inequality in (26) is not satisfied, a trimmed set of index $\mathcal{K} \backslash\left\{\pi_{U}, \ldots, \pi_{K}\right\}$ is obtained. This set is then further trimmed by removing the user index for which the inequality $\mathcal{C}_{\pi_{U}}^{\star}>0$ or $p_{\pi_{U}}^{\star}>0$ does not hold. Overall, our simple procedure for optimizing the unconstrained or constrained EE in the singlecell scenario is summarized below in Algorithm 1.

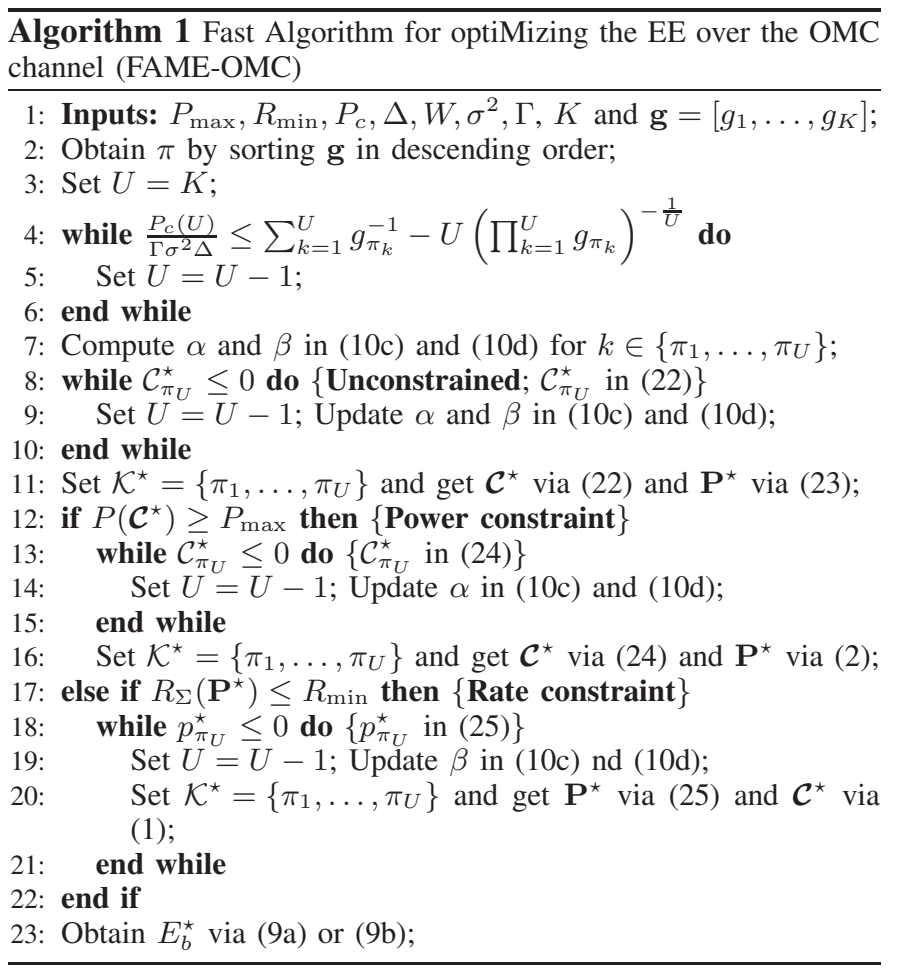




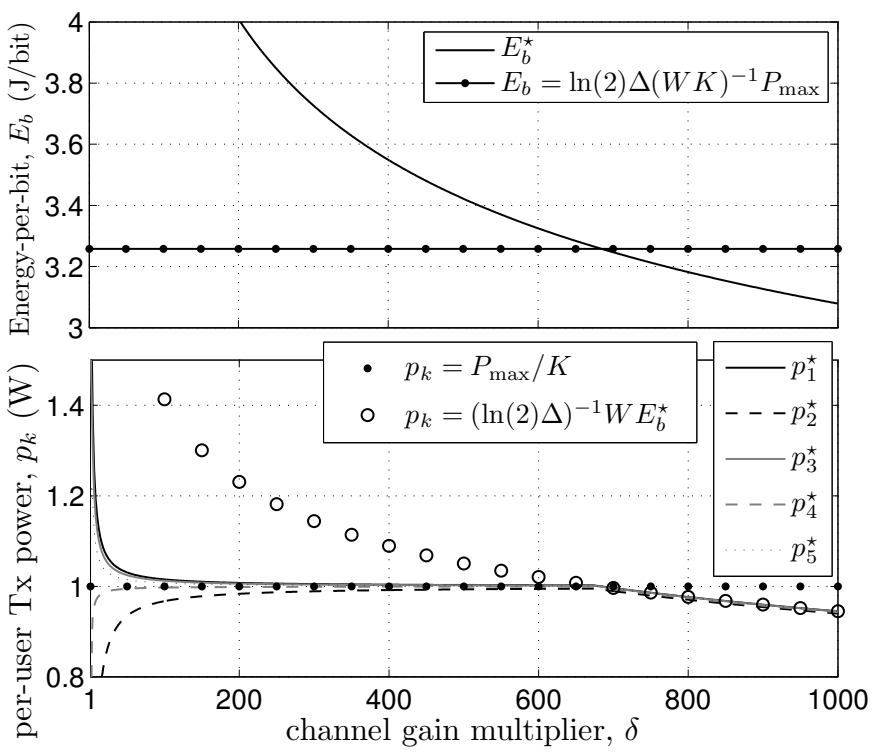

Fig. 2. Optimal energy-per-bit consumption and per-user power as a function of the channel gain multiplier $\delta$.

6) EE optimization insights: As far as the optimization of the sum-rate over the OMC is concerned, it is well-known that the optimal SE-based power allocation strategy is obtained via water-filling [10] such that $p_{k}^{\star}$ is given as in (16). Hence, the most spectral efficient power allocation is equal power allocation when $\Gamma \sigma^{2} g_{k}^{-1} \ll 1$, i.e. when the channel gain-tonoise ratio is high, such that $p_{k}^{\star}=\frac{P_{\max }}{K}$. Similarly, inserting (21) in (2), it turns out that

$$
p_{k}^{\star}=\left[(\ln (2) \Delta)^{-1} W E_{b}^{\star}-\Gamma \sigma^{2} g_{k}^{-1}\right]_{+},
$$

which clearly indicates that equal power allocation is also the most energy efficient power allocation when $\Gamma \sigma^{2} g_{k}^{-1} \ll 1$, such that $p_{k}^{\star}=(\ln (2) \Delta)^{-1} W E_{b}^{\star}$, which reverts to $p_{k}^{\star}=\frac{P_{\max }}{K}$ when $E_{b}^{\star} \geq \ln (2) \Delta(W K)^{-1} P_{\max }$.

In Fig. 2, we depict the optimal energy-per-bit and per-user transmit power for $P_{\mathrm{c}}=130 \mathrm{~W}, \Delta=4.7, P_{\max }=5 \mathrm{~W}, N_{0}=$ $W=1, \Gamma=1, K=5$ users, and the channel gain values $\mathbf{g}=$ $\delta[5,0.2,2,0.5,1]$, where $\delta$ varies from 1 to 1000 . In the lower part of the graph, it can clearly be seen that as $\delta$ increases, or equivalently as the channel gain-to-noise ratio increases (since $\sigma^{2}$ is fixed), as the optimal per-user power allocation converges first towards $p_{k}^{\star}=\frac{P_{\max }}{K}$ for $\delta$ up to 680 and then towards $p_{k}^{\star}=$ $(\ln (2) \Delta)^{-1} W E_{b}^{\star}$ thereafter. The transition begins when $E_{b}^{\star}$ becomes lower than $\ln (2) \Delta(W K)^{-1} P_{\max }$ in the upper part of the graph. Thus, it is in line with our analysis and confirms that equal power allocation is the most energy efficient power allocation over the OMC when the channel gain-to-noise ratio is high.

\section{B. Multi-sector EE optimization}

In the previous subsection, we assumed that no inter-sector interferences occur. In a realistic cellular system, interferences do occur between sectors of different BSs and one practical way to mitigate them is coordination [20]. In the cellular layout of Fig. 1, any given user is surrounded by at least three BSs, i.e. its own serving BS and two closest neighboring BSs. As a result, within the perimeter delimited by the thick black line in Fig. 1, i.e. a cluster of three sectors of three different BSs, the main bulk of the downlink interferences that are experienced by a UE in one of the sectors will arise from its two closest non-serving BSs. In order to improve the EE of cellular systems but with limited feedback, i.e. when assuming that each serving BS has some knowledge about the channel between itself and its own users as well as between the two other non-serving BSs and its own users, we develop a low-complexity energy-efficient optimization algorithm for coordinating the resource allocation within a cluster of three adjacent sectors. Note that the pattern in Fig. 1 can be repeated over the whole cellular system and, without loss of generality, we discuss the coordinated EE optimization for one of this cluster.

Numbering the light, medium and dark grey colored sectors within the cluster of adjacent sectors as sector 1 of BS 1 , sector 2 of BS 2 and sector 3 of BS 3, respectively, the interferences that are generated by the non-serving BSs towards the $k$-users of sector $m$ can be formulated as

$$
I_{m, k}=\sum_{i=1, i \neq m}^{M} \mathbf{P}_{i} \mathbf{1}^{T} g_{m, k}^{i},
$$

where $M=3$. Furthermore, we assume that users are uniformly distributed (UD) within each sector and each sector has the same number of users. As this number goes to infinity, as the likelihood of having users in different sectors with similar relative positions towards their serving $\mathrm{BS}$ and the two non-serving BSs increases, such that $d_{1, k}^{1} \simeq d_{2, k}^{2} \simeq d_{3, k}^{3}$, $d_{2, k}^{1} \simeq d_{3, k}^{2} \simeq d_{1, k}^{3}$ and $d_{3, k}^{1} \simeq d_{1, k}^{2} \simeq d_{2, k}^{3}$. Thus, it is expected that the $k$-th user channel gain of each sector asymptotically verifies $g_{1, k}^{1} \simeq g_{2, k}^{2} \simeq g_{3, k}^{3}, g_{2, k}^{1} \simeq g_{3, k}^{2} \simeq g_{1, k}^{3}$ and $g_{3, k}^{1} \simeq g_{1, k}^{2} \simeq g_{2, k}^{3}$ when considering a distance dependent path-loss for $g_{m, k}^{i}$. In this symmetric scenario, it then makes sense in terms of fairness to allocate the same amount of transmit power to each BS, such that (28) simplifies to

$$
I_{k}=\mathbf{P} \mathbf{1}^{T} G_{k}
$$

where $\mathbf{P}=\mathbf{P}_{1}$ and $G_{k}=\sum_{i=2}^{M} g_{1, k}^{i}$. Using this assumption, $P(\mathcal{C})$ in $(3 \mathrm{a})$ can be rewritten as

$$
P(\mathcal{C})=\mathbf{P} 1^{T}=\frac{\Gamma \sum_{k=1}^{K}\left(2^{\mathcal{C}_{k}}-1\right) g_{k}^{-1} \sigma^{2}}{1-\Gamma \sum_{k=1}^{K}\left(2^{\mathcal{C}_{k}}-1\right) g_{k}^{-1} G_{k}}
$$

by inserting (29) in (3a). Because of the perfect symmetry, the interference that is experienced by user $k$ of each sector will be identical. Thus, optimizing the transmit power and rate of one of the sectors will at the same time optimize the resource allocation of the two other sectors. However, the limitation of using (28) instead of (29) for modeling the intersector interference is that the equality in (29) only holds if the system is perfectly symmetric in terms of channel gains, which can only be the case in theory; in practice (29) is only an approximation of (28), which is likely to be accurate for large numbers of UD users in each sector, making the optimization method suboptimal. By contrast, the major advantage of (29) over (28) is that it greatly simplifies the optimization problem. 
Indeed, (29) satisfies assumption 2) of Theorem I and, hence, the EE optimization framework of Section III can be directly applied to solve this optimization problem in a low-complexity manner as long as the convexity condition also holds.

1) Convexity of $E_{b}$ : Inserting (30) into (8a), the per-sector $E_{b}$ can be re-formulated as

$$
E_{b}(\mathcal{C})=A[f(\mathcal{C})+B[h(\mathcal{C})-1]] g(\mathcal{C})^{-1} h(\mathcal{C})^{-1}
$$

in the coordinated multi-cell scenario, where $h(\mathcal{C})=1-$ $\Gamma \sum_{k=1}^{K}\left(2^{\mathcal{C}_{k}}-1\right) g_{k}^{-1} G_{k}$ and $f(\mathcal{C})$ as well as $g(\mathcal{C})$ are given in (20). It can been shown (see proof the Appendix) that both $f(\mathcal{C})+B[h(\mathcal{C})-1]$ and $h(\mathcal{C})^{-1}$ are log-convex functions and since their product is also log-convex, we can conclude that $E_{b}$ in (31) is log-convex and, hence, convex, as long as $\mathcal{C} \succeq 0$, $\mathcal{C}_{l}>0, h(\mathcal{C})>0, f(\mathcal{C})+B[h(\mathcal{C})-1]>0$ and $P_{c} \geq \Delta \alpha$.

2) Unconstrained EE optimization: Inserting (12) into (10a), we can express $\mathcal{C}_{l}^{\star}$ solely as a function of the EE-optimal transmit power $P\left(\mathcal{C}^{\star}\right)=\mathbf{P}^{\star} \mathbf{1}^{T}=P^{\star}$ such that

$$
\mathcal{C}_{l}^{\star}=\log _{2}\left(P^{\star}+\alpha\left(P^{\star}\right)\right)-\log _{2}\left(K^{\star} \Gamma g_{l}^{-1}\left(\sigma^{2}+P^{\star} G_{l}\right)\right) .
$$

Inserting (32) into (9a), the latter can be re-expressed solely as a function of $P^{\star}$ such that

$$
E_{b}^{\star}=E_{b}\left(P^{\star}\right)=\frac{\Delta P^{\star}+P_{\mathrm{c}}^{\star}}{W\left[K^{\star} \log _{2}\left(\frac{P^{\star}+\alpha\left(P^{\star}\right)}{K^{\star}}\right)-\beta\left(P^{\star}\right)\right]},
$$

where $\alpha\left(P^{\star}\right)=\Gamma \sum_{k \in \mathcal{K}^{\star}} g_{k}^{-1}\left(\sigma^{2}+P^{\star} G_{k}\right)$ and $\beta\left(P^{\star}\right)=$ $\sum_{k \in \mathcal{K}^{\star}} \log _{2}\left(\Gamma g_{k}^{-1}\left(\sigma^{2}+P^{\star} G_{k}\right)\right)$ according to (10c), (10d) and (29). The problem of finding $P^{\star}$ can then be simply solved by using a modified line search algorithm knowing that $\frac{\partial E_{b}\left(P=P^{\star}\right)}{\partial P}=0$ (see Algorithm 2).

3) Constrained EE optimization: The optimal powerconstrained EE can be straightforwardly obtained by inserting $P^{\star}=P_{\max }$ in (33); whereas, in the rate-constrained case

$$
R_{\min }=W\left[K^{\star} \log _{2}\left(\frac{P^{\star}+\alpha\left(P^{\star}\right)}{K^{\star}}\right)-\beta\left(P^{\star}\right)\right],
$$

and, thus, a simple line search on $P^{\star}$ can be performed for solving (34). Then, the optimal rate-constrained EE can be directly obtained by inserting the result of (34) into (33).

4) Practical considerations for coordinated EE optimization: The perfect symmetric scenario that has previously been described for simplifying the coordinated multi-cell EE optimization is not realistic. However, in a realistic scenario, user ordering and grouping can be used at the BS for mimicking this scenario. For instance, each BS can order their users in descending order according to the amplitude of their channel gains and, then, the average user channel and interferer gains can be computed as

$$
\widetilde{g}_{\pi_{k}}=\frac{1}{3} \sum_{m=1}^{3} g_{m, \pi_{k}}^{m} \text { and } \widetilde{G}_{\pi_{k}}=\frac{1}{3} \sum_{m=1}^{3} \sum_{n=1, n \neq m}^{3} g_{m, \pi_{k}}^{n}
$$

Next, $g_{k}$ and $G_{k}$ can be replaced by $\widetilde{g}_{\pi_{k}}$ and $\widetilde{G}_{\pi_{k}}$ in (30) for optimizing the EE. Using averaged instead of actual channel gains is clearly suboptimal, but it becomes more reliable as the number of users increases, as it is indicated in Fig. 3.

In order to show the reliability of our multi-cell EE optimization algorithm, i.e. the Fast Algorithm for optiMizing the
Algorithm 2 Fast Algorithm for optiMizing the EE in the Coordinated Multi-Cell scenario (FAME-CMC)

1: Inputs: $P_{\max }, R_{\min }, P_{c}, \Delta, W, \sigma^{2}, \Gamma, K, \mathbf{g}=\left[g_{1}, \ldots, g_{K}\right]$ and $\mathbf{G}=\left[G_{1}, \ldots, G_{K}\right]$

2: Compute $\widetilde{g}_{k}$ and $\widetilde{G}_{k}$ by using (35) for any $k \in \mathcal{K}$;

3: Obtain $\pi$ by sorting $\left\{\widetilde{g}_{k} / \widetilde{G}_{k}\right\}$ in descending order;

4: Set $\epsilon=10^{-6}$ and $P^{\star}=\epsilon$;

5: Compute $\left[\mathcal{C}_{\pi_{k}}^{\star}\right]_{+}$in (32) for any $k \in\left\{\pi_{1}, \ldots, \pi_{K}\right\}$;

6: Set $U=K-\sum_{k=1}^{K}\left(\mathcal{C}_{\pi_{k}}^{\star}==0\right)$; Update $\alpha\left(P^{\star}\right)$ and $\beta\left(P^{\star}\right)$;

7: Compute $F\left(P^{\star}, U\right)=U \ln \left(\frac{P^{\star}+\alpha\left(P^{\star}\right)}{U}\right)-\beta\left(P^{\star}\right)+$ $\left(P^{\star}+\frac{P_{c}}{\Delta}\right)\left[-U\left(\frac{1+\Gamma \sum_{k=1}^{U} \widetilde{g}_{\pi_{k}}^{-1} \widetilde{G}_{\pi_{k}}}{P^{\star}+\alpha\left(P^{\star}\right)}\right)+\sum_{k=1}^{U} \frac{\widetilde{G}_{\pi_{k}}}{P^{\star} \widetilde{G}_{\pi_{k}}+\sigma^{2}}\right] ;$

8: while $|F|>\epsilon$ do

9: Compute $d F\left(P^{\star}, U\right) \quad=\quad\left(P^{\star}+\frac{P_{c}}{\Delta}\right)$ $\times\left[U\left(\frac{1+\Gamma \sum_{k=1}^{U} \widetilde{g}_{\pi_{k}}^{-1} \widetilde{G}_{\pi_{k}}}{P^{\star}+\alpha\left(P^{\star}\right)}\right)^{2}-\sum_{k=1}^{U}\left(\frac{\widetilde{G}_{\pi_{k}}}{P^{\star} \widetilde{G}_{\pi_{k}}+\sigma^{2}}\right)^{2}\right] ;$

10: $\quad$ Set $P^{\star}=\max \left\{\min \left\{P^{\star}-F / d F, P_{\max }\right\}, \epsilon\right\}$; Update $\alpha\left(P^{\star}\right)$;

11: Compute $\left[\mathcal{C}_{\pi_{k}}^{\star}\right]_{+}$in (32) for $k \in\left\{\pi_{1}, \ldots, \pi_{U}\right\}$;

12: while $\left[\sum_{k=1}^{U}\left(\mathcal{C}_{\pi_{k}}^{\star}==0\right)\right]==0 \& U<K$ do

13: $\quad$ Set $U=U+1$; Update $\alpha\left(P^{\star}\right)$;

Compute $\left[\mathcal{C}_{\pi_{k}}^{\star}\right]_{+}$in (32) for $k \in\left\{\pi_{1}, \ldots, \pi_{U}\right\}$;

end while

Set $U=U-\sum_{k=1}^{U}\left(\mathcal{C}_{\pi_{k}}^{\star}==0\right)$; Update $\alpha\left(P^{\star}\right)$ and $\beta\left(P^{\star}\right)$;

Compute $F\left(P^{\star}, U\right)$ (as given in Step 7:)

\section{end while}

9: if $P^{\star} \geq P_{\max }$ then $\{$ Power constraint

Set $P^{\star}=P_{\max }$ and $U=K$; Update $\alpha\left(P^{\star}\right)$;

while $\mathcal{C}_{\pi_{U}}^{\star} \leq 0$ do $\left\{\mathcal{C}_{\pi_{U}}^{\star}\right.$ in (32) $\}$

Set $U=U-1$; Update $\alpha\left(P^{\star}\right)$;

\section{end while}

24: else if $R_{\Sigma}\left(\mathbf{P}^{\star}\right) \leq R_{\min }$ then $\{$ Rate constraint

25: $\quad$ Set $P^{\star}=P_{\max }$;

26: Do Steps 5: to 17: but with $F\left(P^{\star}, U\right)=$ $U \log _{2}\left(\frac{P^{\star}+\alpha\left(P^{\star}\right)}{U}\right)-\beta\left(P^{\star}\right)-\frac{R_{\min }}{W}$ and $d F\left(P^{\star}, U\right)=U\left(\frac{1+\Gamma \sum_{k=1}^{U} \widetilde{g}_{\pi_{k}}^{-1} \widetilde{G}_{\pi_{k}}}{P^{\star}+\alpha\left(P^{\star}\right)}\right)-\sum_{k=1}^{U} \frac{\widetilde{G}_{\pi_{k}}}{P^{\star} \widetilde{G}_{\pi_{k}}+\sigma^{2}} ;$

\section{7: end if}

28: Obtain $E_{b}^{\star}$ via (33);

29: Set $\mathcal{K}^{\star}=\left\{\pi_{1}, \ldots, \pi_{U}\right\}$ and obtain $\mathcal{C}^{\star}$ via (32) and $\mathbf{P}^{\star}$ via (3);

$\mathrm{EE}$ in the Coordinated Multi-Cell scenario (FAME-CMC) in Algorithm 2, we compare in Fig. 3 the per-sector $E_{b}$ values returned by our FAME-CMC algorithm and the Matlab "fmincon" function averaged over 1000 runs against the number of users in symmetric and non-symmetric (uniform) channel gain conditions. In the symmetric scenario, we deliberately set $g_{1, k}^{1}=g_{2, k}^{2}=g_{3, k}^{3}, g_{2, k}^{1}=g_{3, k}^{2}=g_{1, k}^{3}$ and $g_{3, k}^{1}=g_{1, k}^{2}=g_{2, k}^{3}$ for any $k$ user. We rely on the power and system model parameters of Table $\mathrm{I}$ and set $\Gamma=1$ as well as $\varsigma=0.5$ for plotting these graphs, where the path-loss dependent channel gain between the $v(m)$-th $\mathrm{BS}$ and the $k$-th user of sector $m$ is expressed as

$$
g_{m, k}^{v(m)}=10\left(G_{\mathrm{TxRx}}-\mathrm{PL}\left(d_{m, k}^{v(m)}\right)\right) / 10 .
$$

In (36), $G_{\mathrm{TxRx}}$ is the antenna gain of the BS-UE transmission and $\mathrm{PL}(d)=\mathrm{Pb}_{\mathrm{LOS}}(d) \mathrm{PL}_{\mathrm{LOS}}(d)+\left(1-\mathrm{Pb}_{\mathrm{LOS}}\right) \mathrm{PL}_{\mathrm{NLOS}}(d)$ is the distance dependent path-loss function. In addition, $\mathrm{PL}_{\mathrm{LOS}}(d)$ and $\operatorname{PL}_{\mathrm{NLOS}}(d)$ are the LOS and non-LOS (NLOS) path-loss functions, respectively, and $\mathrm{Pb}_{\mathrm{LOS}}$ is the line-of-sight (LOS) probability, whose values can be found in Table 27 of [32]. 
TABLE I

SIMULATION PARAMETER VALUES

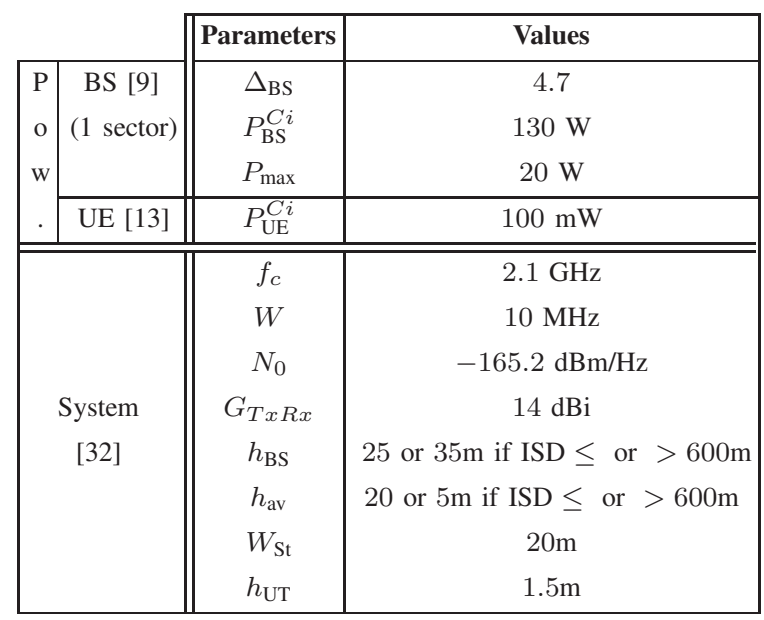
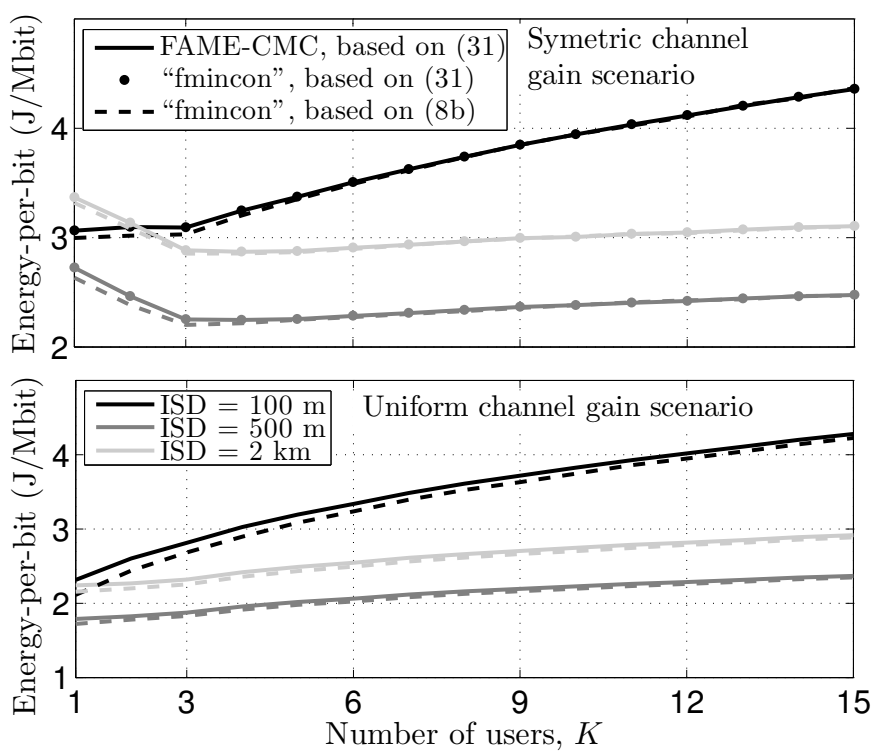

Fig. 3. Per-sector unconstrained $E_{b}$ vs. the number of users in symmetric and uniform channel gain conditions.

Note that we considered here the urban macro (UMa) setting in Table 27 of [32]. The results of our FAME-CMC algorithm and "fmincon" based on (31) in the upper part of Fig. 3 tightly match each other, which numerically confirms the reliability of our algorithm as well as the convexity of (31). We also compare our algorithm with "fmincon" based on (8b) and (28) with a Jain's fairness constraint [33] of $2 / 3$ in both the symmetric and non-symmetric channel gain scenarios in the upper and lower parts of Fig. 3, respectively. The results confirm the sub-optimality of our algorithm; "fmincon" returns lower $E_{b}$ values than our algorithm, however, the performance gap between the two algorithms decreases with the number of users. Thus, in line with our expectation, the reliability of our FAME-CMC improves with the number of users.

\section{Numerical Results AND Discussions}

In order to demonstrate the $\mathrm{EE}$ of our coordinated multicell approach, we benchmark it against the non-coordinated and orthogonal resource allocation (RA) approaches within a cluster of three adjacent cells (see Fig. 1). In the noncoordinated approach, i.e. full reuse case, we consider that all the three sectors share the same bandwidth $W$ and allocate their resources by using the single-cell optimization of Section IV-A, i.e. the Fast Algorithm for optiMizing the EE over the OMC channel (FAME-OMC), without being aware of interferences from other cells. In the coordinated approach, again all the three sectors share the same bandwidth $W$, but resources are allocated by using the multi-cell optimization method of Section IV-B, i.e. FAME-CMC, such that interferences from other cells are taken into account. Finally, in the orthogonal approach, i.e. no reuse case, each sector has a bandwidth of $W / 3$ to avoid any inter-cell interference and the resource is allocated on a per-sector basis via FAME-OMC. We rely here on the same power and system model parameters as in Fig. 3 (see Table I). Note that the extra energy consumption due to the coordination process has not been included in our power model. Our results, which have been obtained through MonteCarlo simulations, depict the per-sector values of the energy consumption, sum-rate and BS transmit power averaged over 10000 runs for each of the three RA approaches.

Intuitively, coordination has a great potential for power and energy savings; indeed, reducing transmit power will reduce interferences, which in turn will counterbalance the loss of rate due to power reduction and, hence, decrease the energy consumption. For instance, let us assume that each sector has one user, i.e. $K=1$, that is placed in the middle of the sector in Fig. 1 such that the distance of the user to its serving BS is $d_{m, 1}^{m}=\mathrm{ISD} / 3$ and to its two closest BSs is $d_{m, 1}^{\tau(m)}=$ $2 \mathrm{ISD} / 3$ and $d_{m, 1}^{\tau(m+1)}=\sqrt{(\sqrt{3} \mathrm{ISD} / 2)^{2}+(\mathrm{ISD} / 6)^{2}}$ for any $m \in\{1,2,3\}$, where $\tau(m)=m \bmod \{3\}+1, \bmod \{$.$\} is$ the modulo operator and ISD stands for inter-site distance, the distance between BSs. Relying on the pathloss model described in (36) and the values of Table I, these distances correspond to the following channel gains of $g_{m, 1}^{m} \simeq 10^{-9}$, $g_{m, 1}^{\tau(m)} \simeq 5.10^{-11}$ and $g_{m, 1}^{\tau(m+1)} \simeq 10^{-11}$ for an ISD of $500 \mathrm{~m}$; whereas, the noise power is such that $\sigma^{2}=3.10^{-13}$. Inserting these values in (1) for $p_{1,1}=p_{2,1}=p_{3,1}=P=20 \mathrm{~W}$, indicates that transmitting at full power provides a maximum $\mathrm{SE}$ of $4.14 \mathrm{bit} / \mathrm{s} / \mathrm{Hz}$ per sector with a total consumed power of about $224 \mathrm{~W}$; whereas, transmitting at $P=0.1 \mathrm{~W}$ gives a $\mathrm{SE}$ of $4.08 \mathrm{bit} / \mathrm{s} / \mathrm{Hz}$ per sector with a total consumed power of about $130.5 \mathrm{~W}$. Hence, the energy consumption at full power is about $54 \mathrm{~J} / \mathrm{bit} / \mathrm{Hz}$, whereas the energy effort at $P=0.1$ $\mathrm{W}$ is about $32 \mathrm{~J} / \mathrm{bit} / \mathrm{Hz}$. Thus, a reduction of $40 \%$ in energy consumption can be achieved through coordination by willing to trade-off $2 \%$ of the SE in this simple example.

In Fig. 4, we compare the three RA approaches that are described above as a function of the ISD for $K=10$ users per sector. Undoubtedly, coordination is really effective for reducing the transmit power at the BS; in this scenario, the latter is reduced by at least $77 \%$ and more than $90 \%$ for small and large ISDs, respectively, in comparison with the orthogonal and non-coordinated approaches. In terms of sumrate, it can be noted that the coordinated approach has a small advantage over the non-coordinated scheme and that the orthogonal method performs better for small to average 


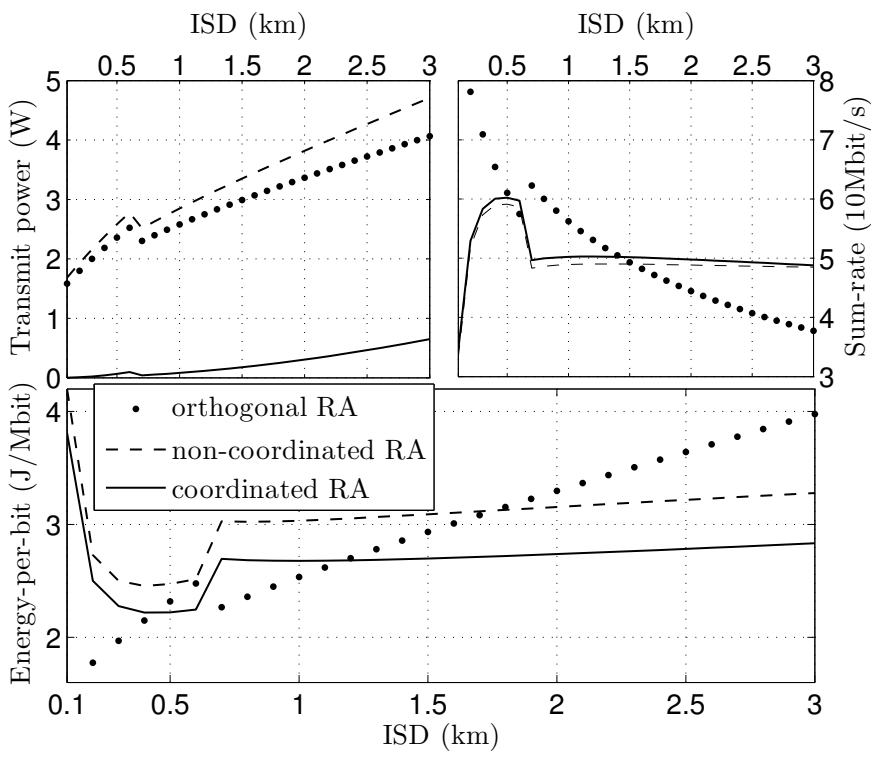

Fig. 4. Comparison of the non-coordinated, coordinated and orthogonal RA approaches in terms of the per-sector transmit power, sum-rate and energyper-bit consumption vs. ISD for $K=10$.

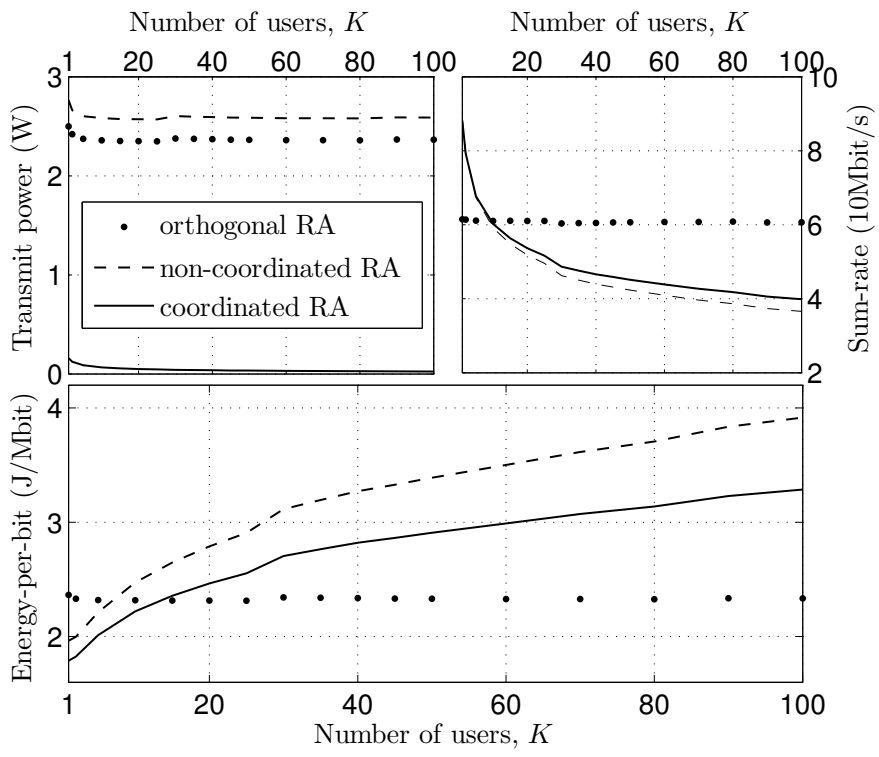

Fig. 5. Comparison of the non-coordinated, coordinated and orthogonal RA approaches in terms of the per-sector transmit power, sum-rate and energyper-bit consumption vs. the number of users for ISD $=500 \mathrm{~m}$.

ISDs. The smaller the cell is, the stronger is the interference from other neighboring cells and, thus, avoiding interference is preferable in that case. Having a very good transmit power reduction capability and edging the non-cooperative approach in terms of sum-rate, the coordinated RA method reduces the energy consumption by 10 to $15 \%$ in comparison with the latter. The coordinated approach outperforms as well the orthogonal approach in terms of $E_{b}$ for ISDs around $500 \mathrm{~m}$ as well as above $1.1 \mathrm{~km}$. Note that the distortion in the shape of the curves occurring at an ISD of around $650 \mathrm{~m}$ is due to a breakpoint in the path-loss model.

In Fig. 5, we compare the same approaches as a function of the number of users per sector for ISD $=500 \mathrm{~m}$. The

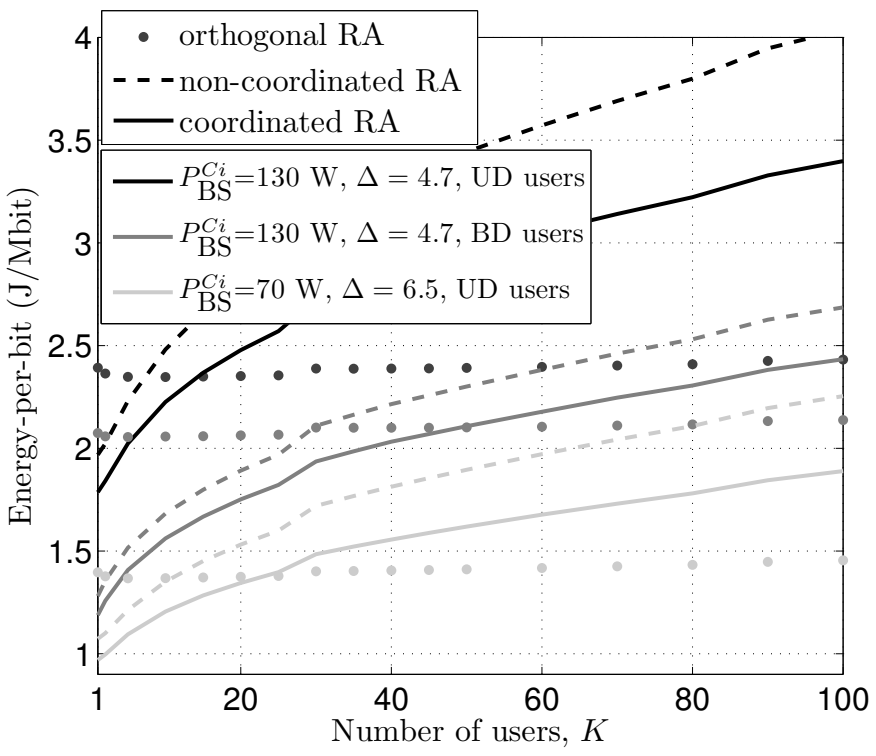

Fig. 6. Comparison of the non-coordinated, coordinated and orthogonal RA approaches in terms of the per-sector energy-per-bit consumption vs. the number of users for ISD $=500 \mathrm{~m}$ and two different power model values as well as user distributions.

results first indicate that our coordinated approach is again really effective for reducing the transmit power at the BS and that it always outperforms the non-coordinated approach for any of the three metrics. In this particular system parameters' setting, the noise power is in the order of $(1 / K) \cdot 10^{-13}$ $\mathrm{W}$, whereas the amplitude of the channel gain of a user near the cell edge, i.e. at $d_{k}=\mathrm{ISD} / 2 \mathrm{~m}$, is in the order of $10^{-10}$. Thus, $\Gamma \sigma^{2} g_{k}^{-1} \ll 1$ in this setting and, hence, equal power allocation is the EE-optimal power allocation for the orthogonal approach according to (27). Consequently, we expect that $p_{k}^{\star} \simeq(\ln (2) \Delta)^{-1}(W /(3 K)) E_{b}^{\star}$ and $R_{\Sigma} \simeq$ $\frac{W}{3 K} \sum_{k=1}^{K} \log _{2}\left(1+g_{k}\left(\ln (2) \Delta \Gamma N_{0}\right)^{-1} E_{b}^{\star}\right)$ such that the persector total transmit power, sum-rate and energy-per-bit are near-invariant with $K$ in the orthogonal method when the users are UD. Whereas, extra users implies extra interferences for both the non-coordinated and coordinated approaches such that their sum-rate and $E_{b}$ performances degrade with the number of users. As a result, the coordinated approach is only more energy efficient than the orthogonal approach for up to $K=15$ users. It can also be remarked that as $K$ increases as the gap between the non-coordinated and coordinated approaches increases, which graphically confirms that the reliability of our approach improves with the number of users.

In Fig. 6, we assess the effects of user distributions and power parameter values on the energy-per-bit performance of the same three approaches for ISD $=500 \mathrm{~m}$. In addition to the UD, we introduce a biased distribution (BD) of users where all the users in a sector are uniformly distributed within a radius of ISD/3 instead of a radius of ISD/2 for UD. In other words, more users are closer to their serving BS in BD. Consequently, a lower energy consumption is achieved by all the three approaches when using BD rather than UD. More interestingly, the coordinated approach outperforms the orthogonal scheme for a wider range of users, up to $K=50$, which emphasizes 
that the coordinated approach is more suitable for serving users within a radius of ISD/3 from their own BS rather than cell edge users. Modifying the power parameters such that $\Delta_{\mathrm{BS}}=6.5$ and $P_{\mathrm{BS}}^{C i}=70 \mathrm{~W}$ also increases the range of $K$ values for which the coordinated method outperforms the orthogonal approach. Since the coordinated approach has a very low power consumption, increasing the slope of the power model favors the coordinated scheme over the two other RA approaches in terms of total consumed power and, in turn, this widens the relative $E_{b}$ performance gap between these approaches.

\section{CONCLUSION}

In this paper, a framework for optimizing the $E E$ in the downlink of a planar cellular system over a frequencyselective and block faded channel has been proposed. We have demonstrated that both constrained and unconstrained EE optimization problems can be greatly simplified under the three assumptions of Section III-A, leading us to the design of one simple and optimal EE-based resource allocation algorithm for the single-cell scenario and one low-complexity and suboptimal EE-based algorithm for the coordinated multicell scenario. In the single-cell scenario, we have derived the explicit formulations of the optimal users' power and rate that minimize the energy consumption of the system for the unconstrained as well as power and rate constrained cases. In turn, we have demonstrated that equal power allocation is both the most energy and spectral efficient strategy when the channel gain-to-noise ratio is high. In addition, this framework has helped to comprehend the very nature of the EE; EE optimization is a generalization of both SE and power optimizations such that the global optimal EE solution is the optimal unconstrained EE solution. Enforcing rate or power constraints on EE can provide a $\mathrm{SE}$ or power optimal solution, which is only suboptimal in terms of EE. Using our low-complexity algorithms for comparing the noncoordinated, orthogonal and coordinated multi-cell resource allocation strategies in realistic power and system settings, we have shown that coordination can be a simple and effective method for improving the EE of cellular systems, especially for medium to large cell sizes and when the main bulk of the users are close to their serving BS. Our results indicate that the per-sector energy consumption of the system can be reduced by up to $15 \%$ by using a coordinated instead of a non-coordinated approach. Moreover, the coordinated approach can reduce the transmit power by more than $90 \%$ in comparison with the two other approaches. We understand that our coordinated resource allocation algorithm is suboptimal and, thus, we plan to further improve it in the future. We also plan to refine our power model by taking into account the extra energy consumption due to coordination.

\section{ACKNOWLEDGMENT}

The first author wish to thank his beloved wife for her support and helpful comments in improving the readability of this paper.

\section{APPENDIX}

A. Proof of convexity for $f(\mathcal{C}), h(\mathcal{C})$ and $f(\mathcal{C})+B[h(\mathcal{C})-1]$

Proof: Let $H(\ln (\bar{f}))$ be the Hessian matrix of the natural logarithm of $\bar{f}$ and $\mathbf{z}=\left[z_{1}, z_{2}, \ldots, z_{K}\right], \mathbf{z} \in \mathbb{R}^{K}$. According to the second-order convexity condition in [18], if $H(\ln (\bar{f}))$ exists and $\psi=\bar{f}^{2} \mathbf{z} H(\ln (\bar{f})) \mathbf{z}^{\dagger} \geq 0$, i.e. $H(\ln (\bar{f}))$ is positive semi-definite, then $\bar{f}$ would be log-convex. Considering that $\bar{f}=f$ in $(20), \psi$ can be expressed after simplifications as

$$
\begin{aligned}
\psi & =(-1)^{\zeta} \ln (2)^{2}\left[\sum_{i=1}^{K-1} \sum_{j=i+1}^{K}\left(z_{i}-z_{j}\right)^{2} 2^{\mathcal{C}_{i}+\mathcal{C}_{j}} g_{i}^{-1} g_{j}^{-1} \rho_{i} \rho_{j}\right. \\
& \left.+\left(B-\sum_{i=1}^{K} g_{i}^{-1} \rho_{i}\right) \sum_{i=1}^{K} z_{i}^{2} 2^{\mathcal{C}_{i}} g_{i}^{-1} \rho_{i}\right],
\end{aligned}
$$

where $\rho_{i}=1$ for any $i \in \mathcal{K}$ and $\zeta=0$. Thus $\psi \geq 0$ and, hence, $f$ is log-convex as long as $B \geq \sum_{i=1}^{K} g_{i}^{-1}$, since $f \geq 0$.

Next, considering that $\bar{f}=h^{-1}$ in $(31), \psi$ can be expressed as in (37) but with $\rho_{i}=-\Gamma G_{i}, \zeta=1$ and $B=1$. Given that $P(\mathcal{C}) \geq 0$ in (30), it implies that $h(\mathcal{C}) \geq 0$, i.e. $1+\Gamma \sum_{i=1}^{K} g_{i}^{-1} G_{i} \geq \Gamma \sum_{i=1}^{K} 2^{\mathcal{C}_{i}} g_{i}^{-1} G_{i}$. Inserting the latter inequality in (37), we obtain

$$
\psi \geq \ln (2)^{2} \Gamma^{2}\left(\sum_{i=1}^{K-1} z_{i} 2^{\mathcal{C}_{i}} g_{i}^{-1} G_{i}\right)^{2},
$$

such that $\psi \geq 0$ and, hence, $h^{-1}$ is log-convex as long as $h(\mathcal{C})>0$.

Finally, considering that $\bar{f}=f(\mathcal{C})+B[h(\mathcal{C})-1], \psi$ can be expressed as in (37) but with $\rho_{i}=1-B \Gamma G_{i}$ and $\zeta=0$. Thus, $\bar{f}$ is log-convex as long as $f(\mathcal{C})+B[h(\mathcal{C})-1]>0$ and $B \geq \sum_{i=1}^{K} g_{i}^{-1} \geq \sum_{i=1}^{K} g_{i}^{-1}\left(1-B \Gamma G_{i}\right)$.

\section{REFERENCES}

[1] 3GPP TSG-SA\#50 SP-1008883GPP, 3GPP Work Item Description, "Study on System Enhancements for Energy Efficiency," 3GPP TSGSA, Istanbul, Turkey, Tech. Rep., Dec. 2010, agreed Work Item 500037 (Release 11).

[2] H. Zhang et al., "Energy efficiency in communications," IEEE Commun. Mag., vol. 48, no. 11, pp. 48-79, Nov. 2010.

[3] —_ "Energy efficiency in communications: part II," IEEE Commun. Mag., vol. 49, no. 6, pp. 28-87, Jun. 2011.

[4] —- "Energy efficiency in communications: part III," IEEE Commun. Mag., vol. 49, no. 8, pp. 52-109, Aug. 2011.

[5] K. Lahiri, A. Raghunathan, S. Dey, and D. Panigrahi, "Battery-driven System Design: A New Frontier in Low Power Design," in Proc. Intl. Conf. on VLSI Design, Bangalore, India, Jan. 2002, pp. 261-267.

[6] H. M. Kwon and T. G. Birdsall, "Channel Capacity in Bits per Joule," IEEE J. Ocean. Eng., vol. OE-11, no. 1, pp. 97-99, Jan. 1986.

[7] S. Cui, A. J. Goldsmith, and A. Bahai, "Energy-Efficiency of MIMO and Cooperative MIMO Techniques in Sensor Networks," IEEE J. Sel. Areas Commun., vol. 22, no. 6, pp. 1089-1098, Aug. 2004.

[8] L. M. Correia, D. Zeller, O. Blume, D. Ferling, Y. Jading, I. Godór, G. Auer, and L. V. D. Perre, "Challenges and Enabling Technologies for Energy Aware Mobile Radio Networks," IEEE Commun. Mag., vol. 48, no. 11, pp. 66-72, Nov. 2010.

[9] G. Auer et al., "How Much Energy is Needed to Run a Wireless Network ?" IEEE Wireless Commun., vol. 18, no. 5, pp. 40-49, Oct. 2011.

[10] R. G. Gallager, Information Theory and Reliable Communication. John Wiley \& Sons, Inc., 1968.

[11] D. N. C. Tse and S. V. Hanly, "Multiaccess fading channels. I. Polymatroid structure, optimal resource allocation and throughput capacities," IEEE Trans. Inf. Theory, vol. 44, no. 7, pp. 2796-2815, Nov. 1998. 
[12] F. Meshkati, H. V. Poor, S. C. Schwartz, and N. B. Mandayam, "An Energy-Efficient Approach to Power Control and Receiver Design in Wireless Networks," IEEE Trans. Commun., vol. 5, no. 1, pp. 33063315, Nov. 2006.

[13] G. Miao, N. Himayat, and G. Y. Li, "Energy-Efficient Link Adaptation in Frequency-Selective Channels," IEEE Trans. Commun., vol. 58, no. 2, pp. 545-554, Feb. 2010.

[14] C. Isheden and G. P. Fettweis, "Energy-Efficient Multi-Carrier Link Adaptation with Sum Rate-Dependent Circuit Power," in Proc. IEEE Globecom, Miami, USA, Dec. 2010.

[15] Z. Chong and E. Jorswieck, "Analytical Foundation for Energy Efficiency Optimisation in Cellular Networks with Elastic Traffic," in Proc. MOBILIGHT 2011, Bilao, Spain, May 2011.

[16] G. Miao, N. Himayat, G. Y. Li, and S. Talwar, "Low-Complexity EnergyEfficient Scheduling for Uplink OFDMA," IEEE Trans. Commun., vol. 60, no. 1, pp. 112-120, Jan. 2012.

[17] F. Héliot, M. A. Imran, and R. Tafazolli, "Energy-efficiency based resource allocation for the scalar broadcast channel," in Proc. IEEE WCNC, Paris, France, Apr. 2012.

[18] S. Boyd and L. Vandenberghe, Convex Optimization. Cambridge, UK: Cambridge Univ. Press, 2004.

[19] O. Somekh, O. Simeone, Y. B. Ness, A. M. Haimovich, U. Spagnolini, and S. Shamai, An information theoretic view of distributed antenna processing in cellular systems; Distributed Antenna Systems: Open Architecture for Future Wireless Communications. CRC, Ed. Boca Raton, FL: Auerbach Publications, Jun. 2007.

[20] M. K. Karakayali, G. J. Foschini, and R. A. Valenzuela, "Network coordination for spectrally efficient communications in cellular systems," IEEE Wireless Commun., vol. 13, no. 4, pp. 56-61, Aug. 2006.

[21] L. Chen, W. Chen, X. Zhang, Y. Zhang, C. Xiong, and D. Yang, "Intercell coordinated resource allocation for mobile WiMAX system," in Proc. IEEE WCNC, Budapest, Hungary, Apr. 2009.

[22] G. Li and H. Liu, "Downlink radio resource allocation for multi-cell OFDMA system," IEEE Trans. Wireless Commun., vol. 5, no. 12, pp. 3451-3459, Dec. 2006.

[23] E. Calvo, O. Muñoz, J. Vidal, and A. Agustín, "Downlink coordinated radio resource management in cellular networks with partial CSI," IEEE Trans. Signal Process., vol. 60, no. 3, pp. 1420-1431, Mar. 2012.

[24] F. Héliot, M. A. Imran, and R. Tafazolli, "Energy-efficiency based resource allocation for the orthogonal multi-user channel," in Proc. IEEE VTC-Fall (to appear), Québec city, Canada, Sep. 2012.

[25] P. Viswanath and D. N. C. Tse, "Sum Capacity of the Vector Gaussian Broadcast Channel and Uplink-Downlink Duality," IEEE Trans. Inf. Theory, vol. 49, no. 8, pp. 1912-1921, Aug. 2003.

[26] S. Verdu, "Spectral Efficiency in the Wideband Regime," IEEE Trans. Inf. Theory, vol. 48, no. 6, pp. 1319-1343, Jun. 2002.

[27] A. Fehske, P. Marsch, and G. Fettweis, "Bit per Joule Efficiency of Cooperating Base Stations in Cellular Networks," in Proc. IEEE Globecom Workshops (GC Wkshps), Miami, USA, Dec. 2010.

[28] G. Auer et al., "D2.3: Energy Efficiency Analysis of the Reference Systems, Areas of Improvements and Target Breakdown," INFSO-ICT247733 EARTH (Energy Aware Radio and NeTwork TecHnologies), Tech. Rep., Nov. 2010.

[29] H. Holtkamp, G. Auer, and H. Haas, "On minimizing base station power consumption," in Proc. IEEE VTC-Fall, San Fransisco, USA, Sep. 2011.

[30] P. Skillermark and P. Frenger, "Enhancing energy efficiency in LTE with antenna muting," in Proc. IEEE VTC-Spring, Yokohama, Japan, May 2012.

[31] R. M. Corless, G. H. Gonnet, D. E. G. Hare, D. J. Jeffrey, and D. E. Knuth, "On the LambertW Function," Adv. Comput. Math., vol. 5, pp. 329-359, 1996.

[32] A. Ambrosy et al., "D2.2: Definition and Parameterization of Reference Systems and Scenarios," INFSO-ICT-247733 EARTH (Energy Aware Radio and NeTwork TecHnologies), Tech. Rep., Jun. 2010.

[33] R. Jain, D. M. Chiuand, and W. Hawe, "A Quantitative Measure of Fairness and Discrimination for Resource Allocation in Shared Systems," DEC Research Report, Tech. Rep. TR-301, 1984.

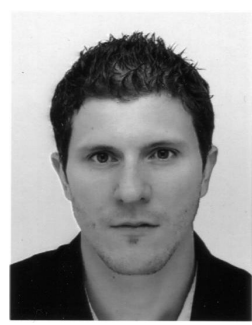

Fabien Héliot (S'05-M'07) received the M.Sc. degree in Telecommunications from the Institut Supérieur de l'Electronique et du Numérique (ISEN), Toulon, France, and the Ph.D. degree in Mobile Telecommunications from King's College London, in 2002 and 2006, respectively. He is currently a research fellow at the Centre for Communication Systems Research (CCSR) of the University of Surrey. He has been actively involved in European Commission funded projects such as FIREWORKS, ROCKET, SMART-Net projects and more recently in the award-winning EARTH project. He is currently involved in the LexNet project, a project investigating human exposure to electromagnetic fields induced by wireless telecommunication networks. His main research interests are energy efficiency, cooperative communication, MIMO, and radio resource management.

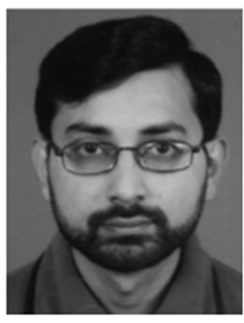

Muhammad Ali Imran (M'03, SM'12) received his B.Sc. degree in Electrical Engineering from University of Engineering and Technology Lahore, Pakistan, in 1999, and the M.Sc. and Ph.D. degrees from Imperial College London, UK, in 2002 and 2007, respectively. He secured first rank in his BSc and a distinction in his MSc degree along with an award of excellence in recognition of his academic achievements, conferred by the President of Pakistan. He is currently a Reader in the Centre for Communication Systems Research (CCSR) at the University of Surrey, UK. He has been actively involved in European Commission funded research projects ROCKET, EARTH, LexNet and iJoin; Mobile VCE funded project on fundamental capacity limits; EPSRC funded India-UK ATC and REDUCE projects. His main research interests include the analysis and modelling of the physical layer, optimization for the energy efficient wireless communication networks and the evaluation of the fundamental capacity limits of wireless networks.

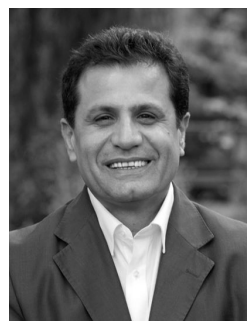

Rahim Tafazolli (SM'09) is a professor and the Director of Centre for Communication Systems Research (CCSR) and 5G Innovation Centre (5GIC), University of Surrey, United Kingdom. He has been active in research for more than 25 years and has authored and co-authored more than 800 papers in refereed international journals and conferences. Professor Tafazolli is a consultant to many mobile companies, has lectured, chaired, and been invited as keynote speaker to a number of IET and IEEE workshops and conferences. He has been Technical Advisor to many mobile companies, all in the field of mobile communications. $\mathrm{He}$ is the Founder and past Chairman of IET International Conference on 3rd Generation Mobile Communications. He is a Fellow of the IET and WWRF (Wireless World Research Forum). He is Chairman of EU Expert Group on Net!Works Technology Platform. 